CANCER SUSCEPTIBILITY

\title{
Location, location, location...
}

Sporadic cancers are known to have a significant hereditary component, but the cancer-susceptibility genes that are involved are hard to locate. However, this might be about to change; Peter Demant and colleagues, reporting in the July issue of Nature Genetics, have used a genetic approach to identify a candidate susceptibility gene that is deleted in human cancer and maps to the susceptibility to colon cancer $(S c c 1)$ locus in mice.

The authors crossed two mouse strains that carry different alleles for the $S c c 1$ locus - one that conferred resistance to colon cancer, and the other that conferred susceptibility. Different markers surround the allele in these two strains, allowing the identification of offspring in which this region had undergone recombination. By correlating the recombinant map with cancer susceptibility, the precise area that contains $S c c 1$ was located. A single gene, Ptprj, is contained within this region, and an analysis of the homologous region in humans revealed the same. So, Ptpri is the only candidate gene for $S c c l$.

Ptpri encodes the protein tyrosine phosphatase receptor type J, which has a catalytic domain and eight fibronectin repeats. But what evidence is there that its loss promotes tumorigenesis? Sporadic colorectal adenocarcinomas were screened for loss of heterozygosity in the homologous region in humans, and two linked but independently deleted

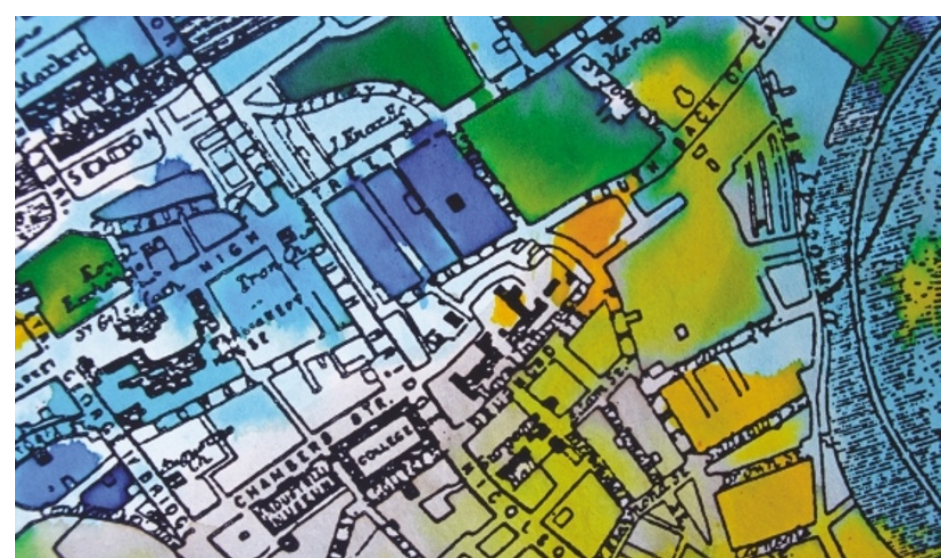

regions were identified - both of these were deleted in $\sim 50 \%$ of cases. One of them contained PTPRJ in the smallest common deleted region, and, in one tumour only, this gene was deleted. Interestingly, loss of heterozygosity was also observed in some lung and breast carcinomas.

The authors also sequenced the $P T P R J$ exons, and discovered seven polymorphisms - one of which, A1176, was preferentially lost in favour of $\mathrm{C} 1176$. This polymorphism occurs within a fibronectin repeat, and is thought to alter the protein conformation, potentially affecting its interaction with other proteins. Only two missense mutations were identified, which indicates that, as with tumour suppressors such as KIP1 and ARF, loss of only one allele is sufficient to provide a growth or survival advantage for cancer cells.

So, by careful mapping in mice, it might be possible to locate more cancer-susceptibility genes. This should shed new light on a previously unexplored area of cancer research.

Emma Greenwood

\section{(Q) References and links} ORIGINAL RESEARCH PAPER Ruivenkamp, C. A. L. et al. Ptprij is a candidate for the mouse colon-cancer susceptibility locus Scc 1 and is frequently deleted in human cancers. Nature Genet. 31, 295-300 (2002)

\section{WEB SITES}

Encyclopedia of Life Sciences:

http://www.els.net colon cancer

Peter Demant's lab:

http://www.nki.nl/research/sr2000/mol-

\section{IN BRIEF}

DRUG RESISTANCE

Smac agonists sensitize for Apo2L/TRAlL- or anticancer drug-induced apoptosis and induce regression of malignant glioma in vivo.

Fulda, S. et al. Nature Med. 15 Jul 2002 (doi:10.1038/nm735)

Most cancer therapeutics induce apoptosis, but tumours can become resistant to this. However, Smac agonists can improve the therapeutic response by sensitizing cells to apoptosis. Smac is released by the mitochondria in response to apoptotic stimuli, and potentiates apoptosis by antagonizing the activity of inhibitor of apoptosis proteins (IAPs). Smac peptides sensitized resistant melanoma and neuroblastoma cells to apoptosis and enhanced the antitumour activity of TRAIL therapy in a xenograft model.

\section{GENOMIC INSTABILITY}

\section{Suppressed CpG mutability and enhanced} tumorigenesis in MBD4-deficient mice.

Millar, C. B. et al. Science 297, 403-405 (2002)

Cytosine-to-thymine transitions occur frequently in the human genome, as the methylated cytosine at a CpG site can be deaminated to form thymine. Many of these mutations are repaired by thymine DNA glycosylases, such as MBD4. So might loss of MBD4 increase mutations and hence tumorigenesis? $M b d 4^{-/}$mice have three times more cytosine-to-thymine transitions, and tumour formation is increased in a cancersusceptible $A p c^{M i n /+}$ background.

\section{CANCER EPIDEMIOLOGY}

\section{Geographical variation in the penetrance of CDKN2A} mutations for melanoma.

Bishop, D. T. et al. J. Nat/ Cancer Inst. 94, 894-903 (2002)

Inherited mutations in the CDKN2A gene - which encodes INK4A and ARF - increase susceptibility to melanoma. An analysis of 80 susceptible families from Europe, the United States and Australia revealed that gender and alterations in ARF did not affect CDKN2A penetrance, but the incidence rate of melanoma in the population did. Whereas the average penetrance increased from 0.3 to 0.67 between the ages of 50 and 80 , the incidence in Australia increased from 0.32 to 0.91 , indicating that the same factors affect population incidence of melanoma and CDKN2A penetrance.

\section{ONCOGENESIS}

Mice deficient in the Rac activator Tiam1 are resistant to Ras-induced skin tumours.

Malliri, A. et al. Nature 417, 867-871 (2002)

Rho proteins are crucial mediators of many aspects of tumorigenesis, so loss of a Rho-protein activator could reduce the incidence of tumour formation. Mice were generated that were deficient for the Rac activator Tiam1. These were resistant to the formation of Ras-induced skin tumours, and the few that did develop grew slowly. Interestingly, these were more malignant than those that developed in a wild-type background. 